\title{
ESTUDIO PSICOLÓGICO SOBRE LOS PATRONES DE CONDUCTA EN CONTEXTOS DE TRÁFICO, EN GRUPOS DE AUTOMOVILISTAS PARTICULARES Y PROFESIONALES DE LIMA METROPOLITANA
}

\author{
Carlos Ponce D. ${ }^{1}$, Mario Bulnes B. ${ }^{2}$, Jaime Aliaga T. ${ }^{3}$, Eliana Delgado C. ${ }^{4}$, Rolando Solis N. ${ }^{5}$
}

(Recibido el 03/07/2006, aceptado el 02/08/2006)

\begin{abstract}
RESUMEN
Objetivos: 1. Conocer los estilos de comportamiento en contextos de tráfico que presentan los automovilistas de servicio público y los choferes particulares de Lima Metropolitana. 2. Detectar la existencia de diferencias significativas en los estilos de conducta en contextos de tráfico, entre los choferes de servicio público y los automovilistas particulares de Lima Metropolitana, de manera global y específica. 3. Investigar la existencia de diferencias significativas en los estilos de conducta en contextos de tráfico entre los conductores de servicio público y los choferes particulares de Lima Metropolitana, en relación a las variables: edad cronológica; estado civil; grado de instrucción; lugar de procedencia; pertenencia del vehículo; la conducción como ocupación principal; tiempo en la conducción; accidentes de tránsito; papeletas recibidas por infracciones; problemas de salud; problemas auditivos; problemas de motricidad; problemas familiares y problemas emocionales. 4. Estudiar la confiabilidad y validez del cuestionario PSS que evalúa patrones de conducta de los automovilistas en contextos de tráfico.
\end{abstract}

Diseño: Es descriptivo-comparativo y en el cual hemos recolectado información relevante en cuatro muestras diferentes de choferes que conducen vehículos en Lima Metropolitana, con respecto a un mismo fenómeno o aspecto de interés (los estilos de comportamiento en contextos de tráfico), y luego hemos caracterizado este fenómeno en base a la comparación de los datos recogidos.

Material y métodos: En el presente estudio se ha trabajado con una muestra de 740 choferes varones que conducen vehículos en Lima Metropolitana, cuyas edades han oscilado entre los 19 y los 69 años, la cual fue seleccionada a través de un muestreo no-probabilístico de tipo intencional o intencionado. Para el presente estudio se han tomado muestras en Empresas de Transportes Urbano e Interprovincial y de Taxistas, así como también a particulares que manejan sus propios vehículos. De los 740 conductores examinados, 249 son automovilistas particulares; y 491 son conductores de servicio público, entre taxistas (152); microbuseros (228) y choferes interprovinciales (111), respectivamente.

\footnotetext{
Docente Principal, Facultad de Psicología de la UNMSM. E-mail: cponced@unmsm.edu.pe

Docente Principal, Facultad de Psicología de la UNMSM. E-mail: mbulnesb@unmsm.edu.pe

Docente Principal, Facultad de Psicología de la UNMSM. E-mail: jaliagat@unmsm.edu.pe

4 Docente Auxiliar, Facultad de Psicología de la UNMSM. E-mail: edelgadoc@unmsm.edu.pe

5 Docente Auxiliar, Facultad de Psicología de la UNMSM. E-mail: rsolisn@unmsm.edu.pe
} 
El instrumento utilizado ha sido el cuestionario de personalidad PSS, el cual forma parte de la BC, Batería de Conductores que procede del Departamento I + D de TEA Ediciones y J. L. Fernández-Seara. La escala tiene 57 ítemes y ha sido estructurada en dos partes, en la primera se estudia la relación rasgos-situaciones en el contexto de tráfico, y en la segunda, el papel de las situaciones de tráfico en la conducta del individuo al volante. A partir de la información recabada de las dos partes de la Escala se pueden obtener dos patrones de conducta o estilos de comportamiento denominados Tipo $\mathrm{S}$ y Tipo $\mathrm{N}$, que se refieren a dos patrones de personalidad a la hora de realizar la conducción en automóvil. El patrón de conducta Tipo S, caracteriza a los sujetos que son seguros, prudentes, responsables, atentos y precavidos (tipo ideal), a diferencia del patrón de conducta Tipo N, que caracteriza a los choferes cuya conducta se manifiesta desajustada, de alto riesgo y peligrosa.

El análisis estadístico se llevó a cabo en tres etapas: análisis descriptivo y exploratorio para describir de manera detallada la distribución de la muestra examinada; análisis psicométrico del PSS; y análisis inferencial para la contrastación de hipótesis. Finalmente, se ha aplicado el Chi cuadrado para efectuar un análisis cualitativo de la predominancia de respuesta en cada uno de los reactivos en virtud a los estilos de comportamiento estudiados.

Conjuntamente con el cuestionario PSS, se ha aplicado una pequeña encuesta de 18 preguntas que constituye el registro de las variables independientes asignadas.

Resultados: En el cuestionario PSS, los resultados no se aproximan a la distribución normal, por lo que se ha trabajado la investigación con estadísticos no-paramétricos sobre la base de rangos. Los resultados corroboran que los ítemes del cuestionario son consistentes entre sí. El análisis de la confiabilidad por consistencia interna nos indica coeficientes alfa razonables en toda la prueba $(\mathrm{S}+\mathrm{N})$, que nos permite inferir la existencia de puntajes confiables. El análisis factorial exploratorio nos revela que existe un solo factor bipolar que para un caso, tiene una varianza que explica el 39,16\% (factor S), y en el otro caso, una varianza que explica el 18,84\% (factor N), los cuales en su conjunto explican el $57,81 \%$ de la varianza total.

Conclusiones: Entre las conclusiones más importantes del presente estudio, tenemos: 1. Los choferes interprovinciales presentan comparativamente estilos de comportamiento positivos en contextos de tráfico que le permiten conducir bajo una percepción y aceptación del riesgo adecuada; son precavidos, atentos y responsables, con alto nivel de seguridad y controlan adecuadamente su conducta, constituyendo el grupo de choferes que marca la diferencia con las otras modalidades examinadas (particulares, microbuseros y taxistas), que por el contrario presentan una conducta desajustada, peligrosa y de alto riesgo. 2. En la muestra total examinada de choferes de servicio público y particulares, predomina el estilo de conducta tipo $\mathrm{N}$, aunque a nivel específico, el tipo $\mathrm{N}$ es el predominante en automovilistas particulares, taxistas y microbuseros, en tanto que el factor $\mathrm{S}$ es el predominante en los choferes interprovinciales. 3. Los choferes que manejan vehículos de propiedad de una Empresa presentan estilos positivos de conducta en contextos de tráfico frente a los que son propietarios o a los que alquilan vehículos; desde el análisis cualitativo se detecta que los choferes que manejan su propio vehículo, así como los que conducen vehículos alquilados, es predominante el tipo N; en tanto que en los choferes que trabajan para Empresas predomina el tipo S. 4. A mayor tiempo en la conducción van mejorando gradualmente los estilos de comportamiento en contextos de tráfico; en tal sentido, el tipo $\mathrm{N}$ predomina entre los conductores que manejan de 1-5 y de 6-10 años, presentándose un equilibrio entre $\mathrm{S}$ y $\mathrm{N}$ entre los que manejan entre 11-15, en tanto que el tipo $\mathrm{S}$ es predominante en los choferes que manejan de 16-20 y de 21 o más años. 5. La frecuencia de accidentes de tránsito en choferes nos revela estilos de comportamiento negativos e inadecuados en contextos de tráfico que se van agudizando con el tiempo, frente a la ausencia de accidentes de grupos de choferes que se expresa en una adecuada conducción. 6. Existen diferencias significativas 
en los estilos de comportamiento globales en contextos de tráfico en razón a la variable problemas familiares, entre los choferes que manifiestan tener problemas familiares, respecto de los que no reportan este tipo de problema, a favor de los segundos; el análisis cualitativo sobre el punto pone de manifiesto que en los choferes que manifiestan problemas familiares es predominante el factor $\mathrm{N}$, observándose un relativo equilibrio entre los tipos $\mathrm{S}$ y $\mathrm{N}$ en los conductores que no refieren dichos problemas.

Palabras clave: Patrones de comportamiento, Contextos de tráfico, Accidentes de tránsito, Cuestionario de personalidad, Automovilistas particulares, Taxistas, Microbuseros y choferes interprovinciales.

\begin{abstract}
Objectives: 1. To understand behaviour styles of private and public service drivers in Lima, Metropolitan Area. 2. To detect the existence of significant differences in behaviour styles in traffic environments, within private and public service drivers of Lima, Metropolitan Area, in a global and specific manner. 3. To investigate the existence of significant differences of behaviour styles in traffic environments, within private and public service drivers of Lima, Metropolitan Area, in relation to the following variables: age, civil status, education level, geographical origin, vehicle property, driving as a main occupation, driving schedule/time length, traffic accidents record, traffic tickets, health problems, hearing problems, motor faculties, family problems and emotional problems. 4. To study the reliability and validity of the PSS questionnaire that evaluates behavior patterns of driver in traffic environments.
\end{abstract}

Outline: It is descriptive-comparative, in which we have collected relevant information in four different samples of drivers in Lima, Metropolitan Area, according to a same phenomenon or aspect of interest (behavior styles in traffic environments), and then we have characterised this phenomenon based on the comparison of the collected data.

Materials and method: In the present study we have worked with a sample of 740 male drivers in Lima, Metropolitan Area, with ages between 19 and 69, which was selected through a non-probabilistic intentional type sampling. For the present study, we have taken samples from urban transportation, interstate transportation and taxicab companies, as well as private drivers that own their vehicles. Out of 740 drivers examined, 249 are private drivers, 491 are public service drivers, divided in taxicab drivers (152), bus drivers (228) and interstate bus drivers (111).

The device we used consisted in the personality questionnaire (PSS), which is part of the BC, Driver's Data» from the R $+\mathrm{D}$ department of TEA Editions and J.L. FernándezSeara. The scale has 157 items and has been structured in two parts; the first one studies the relation between characteristics and situations in traffic environments, the second part studies the role of traffic situations in the driver's behavior.

The information collected from both parts of the scale allows to obtain two behavior patterns or behavior styles called S Type and $\mathrm{N}$ Type, which refer to two personality patterns shown when driving vehicles. Type $\mathrm{S}$ describes self-secure, prudent, responsible, attentive and cautious drivers (ideal type), unlike Type $\mathrm{N}$, which describes unadjusted, high risk and dangerous drivers.

The statistic analysis took place in three stages: Descriptive and exploratory analysis to describe in detail the distribution of the examined sample, psychometric analysis of the PSS, inferential analysis for the hypothesis contrast. Finally, Chi-Square statistic has been used for a qualitative analysis of the answer predominance in each one of the reactive tests applied to the behavior styles studied. 
Along with the PSS questionnaire, a small survey of 18 questions has been taken. The survey consists in the registration of the independent variables assigned.

Results: In the PSS questionnaire, the results do not approximate to the normal distribution, for which this research has worked with non-parametric statistics on the range base. These results prove that the items of the questionnaire are consistent amongst them. The confidence analysis by internal consistency shows us reasonable alpha coefficients in the complete test $(\mathrm{S}+\mathrm{N})$, which allows us to infer the existence of confident scores. The exploratory factorial analysis reveals us that there is only one bipolar factor that, for one case, has a variance that explains $39.16 \%$ ( $\mathrm{S}$ factor) and for the other case has a variance that explains $18.84 \%$ ( $\mathrm{N}$ factor). Both of them together explain $57.81 \%$ of the total variance.

Conclusions: The most important conclusions of the present study are: 1 . The interstate drivers present positive behavior styles in traffic environments that allow them to drive under an adequate risk perception and acceptance; they are careful, attentive and responsible, with a great level of security and an adequate control of their conduct, being part of the group of drivers that draw the line between other types of drivers examined (private, bus drivers and taxicab drivers), who, on the other hand have an unadjusted, dangerous and high risk behaviour. 2 . In the total sample examined, the $\mathrm{N}$ type behavior style predominates, although in a specific level, the $\mathrm{N}$ type is predominant amongst private, taxicab and bus drivers, while the $\mathrm{S}$ factor is predominant amongst interstate drivers. 3 . The drivers with vehicles that are owned by a company have positive behavior styles in traffic environments, in comparison to the ones that own or rent vehicles. In qualitative analysis, $\mathrm{N}$ type is predominant amongst drivers that have their own vehicle and the ones who rent. Meanwhile, the $S$ type is predominant for drivers that work for a particular company. 4. Longer driving times imply gradual improvements in behavior styles in traffic environments. That way, the $\mathrm{N}$ type is predominant amongst drivers that work from 1 to 5 and from 6 to 10 years, showing an equilibrium in $\mathrm{S}$ and $\mathrm{N}$ type when drivers work from 11 to 15 years, while the $\mathrm{S}$ type is predominant amongst drivers that work from 16 to 20 and from 21 or more years. 5 . The frequency of traffic accidents in drivers with negative and inadequate behavior styles in traffic environments increases over the years, while an absence of accidents is observed in groups of drivers with adequate driving. 6. There are significant differences in global behavior styles in traffic environments when the variable «family problems» is observed. Drivers with family problems report better behavior styles than the ones with no family problems. The qualitative analysis shows that the $\mathrm{N}$ factor is predominant amongst drivers that have family problems. In addition, equilibrium between the $\mathrm{S}$ and $\mathrm{N}$ type is observed in drivers that don't have such problems.

Keywords: Behavior Patterns, Traffic Enviroments, Traffic accidents, Personality questionnaire, Private drivers, Taxicab drivers, Bus drivers and interstate bus drivers. 


\section{INTRODUCCIÓN}

El tráfico como fenómeno social de primera magnitud es una realidad que nadie desconoce o discute. El deseo y la necesidad de movilizarse sentidos por la población general y concebidos como un bien social, han inspirado especialmente la cultura de nuestra época con carácter general; pero es en el tráfico vial donde, el conflicto de relaciones que cualquier fenómeno social genera, se percibe con mayor intensidad, porque es en él donde cada una de las personas, desde su nacimiento, es a la vez sujeto activo y pasivo de dicho fenómeno, su beneficiario y eventualmente su víctima.

Desde los primeros tiempos de la motorización, la Psicología y los psicólogos participaron activamente en las propuestas de solución al problema de los accidentes de tráfico. Una de las primeras propuestas de la Psicología se centró en la exploración psicológica y selección de conductores. Muy pronto, la mayor parte de los países avanzados del mundo exigieron, a los conductores profesionales de vehículos, automóviles de servicio público o de transportes colectivos, algún tipo de certificación o «dictamen de capacitación», obtenido a partir de un examen psicotécnico de aptitud para la conducción.

En las últimas décadas del siglo XX y en lo que va del siglo XXI, se ha incrementado considerablemente el parque automotor a nivel mundial, lo cual ha traído como consecuencia nuevos retos y problemas en el ámbito del tráfico, el transporte y la seguridad vial. L. Montoro González (1998) nos dice que los vehículos con motor han sido, son y serán una fuente constante de progreso, de riqueza y de cultura. Nuestra sociedad sería impensable sin ellos y pese a todos los problemas que han generado, los beneficios globales que hemos tenido, las vidas que se han salvado y el desarrollo y el avance que han significado son, con mucho, superiores a los daños ocasionados. Sólo una visión parcial e ingenua podría defender lo contrario.

En 1998, la Organización Mundial de la Salud (OMS) informaba que cada hora que pasa unas 65 personas mueren en el mundo por un siniestro y cerca de 2000 son ingresadas a algún hospital. Carlos B. Rodríguez (2005) reporta que 1,2 millones de personas mueren en el mundo y hasta 50 millones resultan heridas en accidentes de tráfico por año. Los datos son abrumadores y demuestran que las defunciones y lesiones causadas por el tránsito constituyen un problema de salud pública mundial de proporciones endémicas. Según previsiones de la OMS, en los próximos veinticinco años, los accidentes de tráfico, junto con las enfermedades mentales y sus secuelas, serán los dos problemas de salud pública más graves con los que la sociedad se tendrá que enfrentar.

En el Perú, durante los años 1990 al 2000 se registraron 692,848 accidentes de tránsito, los cuales ocasionaron la muerte de 31555 personas y 210313 lesionados. Para el año 2000, la tasa de mortalidad por 10000 vehículos es 27 y la tasa por 100000 habitantes es 12 . Según la clase de accidente, estos se distribuyeron en: choques $(71,71 \%)$; atropellos $(20,44 \%)$; volcaduras (2.48\%); caídas de ocupantes de vehículos $(2,04 \%)$ y otros $(3,33 \%)$. El grupo más afectado fue entre 15 a 65 años $(84 \%)$ y el restante se distribuye en un $9 \%$ para menores de 15 años, y $7 \%$ para mayores de 65 años.

Según la información del Touring Automóvil Club del Perú, las causas de los accidentes de tránsito son: $33 \%$ exceso de velocidad; $28 \%$ imprudencia de los conductores; $12 \%$ imprudencia de los peatones; $11 \%$ conductores en estado de ebriedad y sólo un $6 \%$ falla mecánica o inadecuada señalización. Estadísticas recientes informan que cada año se producen más de 
74000 accidentes con un saldo de 4,000 fallecidos y más de 100000 personas lesionadas, que generan gastos de 63 millones de soles, siendo el Departamento de La Libertad el que puntúa con la más alta tasa a nivel nacional con un $9.6 \%$, y los mototaxistas los que presentan la mayor cantidad de siniestros que corresponden a un $21 \%$ del total. Se precisa además que el $84 \%$ de los accidentes pudieron evitarse tomando medidas preventivas.

La responsabilidad del factor humano y en concreto la buena formación integral del conductor, como un elemento clave para evitar la accidentalidad y mejorar la seguridad vial, aparece en Europa desde la década de 1950, como una nueva necesidad de introducir un cambio en la formación de los choferes, toda vez que los estudios revelan que el error humano está implicado entre el 70 y el $90 \%$ de los accidentes (Montoso, Carbonell, Sanmartín y Tortosa, 1995; Montoso, Carbonell, Tortosa y Sanmartín, 1996). Las causas hay que buscarlas en diversos factores, tales como: causas psíquicas, búsqueda del riesgo, causas físicas, falta de respeto a los reglamentos o normas de circulación, deficiencias en la percepción, entre otras.

En lo que se refiere a evaluar conductores, al clásico modelo de habilidades (psicofísicas), se han sumado nuevos modelos alternativos denominados «propositivos o de riesgo», destinado a un proceso de evaluación-intervención-prevención mucho más efectivo, de los cuales para nuestro estudio hemos elegido el de J.L. Fernández Seara, el cual nos ofrece un instrumento denominado PSS que nos va a proporcionar dos estilos de comportamiento denominados tipo $\mathrm{S}$ y tipo $\mathrm{N}$, que se refieren a dos patrones de personalidad a la hora de realizar la conducción de un vehículo automotriz.

\section{Problema de investigación}

Sobre la base de todo lo señalado anteriormente, el presente estudio exploratorio de tipo descriptivo-comparativo pretende responder a las siguientes interrogantes:

1. ¿Presentan los automovilistas de servicio público (microbuseros, taxistas y choferes interprovinciales) y los conductores particulares de Lima Metropolitana estilos de comportamiento diferenciados en contextos de tráfico?

2. ¿Qué estilos de comportamiento en contextos de tráfico, presentan comparativamente los choferes de servicio público y los automovilistas particulares de Lima Metropolitana en relación a las variables: edad cronológica; estado civil; grado de instrucción; lugar de procedencia; pertenencia del vehículo; la conducción como ocupación principal; tiempo en la conducción; accidentes de tránsito; papeletas recibidas por infracciones; problemas de salud; problemas auditivos; problemas de motricidad; problemas familiares y problemas emocionales?

\section{Objetivos}

1. Estudiar la confiabilidad y validez del cuestionario PSS que evalúa patrones de conducta de los automovilistas en contextos de tráfico.

2. Conocer los estilos de comportamiento en contextos de tráfico que presentan los automovilistas de servicio público y los choferes particulares de Lima Metropolitana. 
3. Detectar la existencia de diferencias significativas en los estilos de conducta en contextos de tráfico, entre los choferes de servicio público y los automovilistas particulares de Lima Metropolitana, de manera global y específica.

4. Investigar la existencia de diferencias significativas en los estilos de conducta en contextos de tráfico entre los conductores de servicio público y los choferes particulares de Lima Metropolitana, en relación a las variables: edad cronológica; estado civil; grado de instrucción; lugar de procedencia; pertenencia del vehículo; la conducción como ocupación principal; tiempo en la conducción; accidentes de tránsito; papeletas recibidas por infracciones; problemas de salud; problemas auditivos; problemas de motricidad; problemas familiares y problemas emocionales.

\section{Marco teórico}

El interés despertado por la psicología del tráfico y de la seguridad vial en otros países y las perspectivas que esta encierra son cada vez más patentes, tanto en la sociedad como en la propia administración que demandan soluciones al grave problema en que se ha convertido la accidentabilidad en las carreteras.

El factor humano, tan presente en la gran mayoría de los accidentes de tránsito, nos hace reflexionar sobre las enormes y variadas posibilidades de investigación y aplicación de esta especialidad en nuestro país. Desde los centros de reconocimiento, los psicólogos realizan una labor selectiva, pero lo que es más importante, creemos, es la labor preventiva e informativa, dado que en la actividad diaria podemos detectar choferes con niveles excesivos de inestabilidad emocional, agresividad, ansiedad, estrés, etc., informando del riesgo que conlleva tal estado, de manera que puedan conducir el mayor número de personas con garantías de seguridad para toda la sociedad.

Los psicólogos que trabajan en materia de tráfico y seguridad vial tienen que estar al día y renovar sus conocimientos en la especialidad. En tal sentido, mencionamos algunos de los problemas más importantes:

1. En relación a los vehículos, asistimos a una permanente sofisticación en los motores y especialmente en la potenciación de todos los mecanismos de seguridad activa y pasiva, es decir aquellos elementos del vehículo que sirven, o bien para evitar que se produzca el accidente o bien para evitar, reducir o minimizar las consecuencias en las personas, tras producirse el siniestro.

Uno de los retos radica en llegar a conocer el impacto que las nuevas tecnologías tienen en el comportamiento del conductor, ya que algunos estudios revelan que las mejoras en los sistemas de seguridad de los vehículos, puede hacer que algunos conductores sean más proclives a los accidentes, porque al tener más sensación de seguridad, «compensaban» las ventajas del sistema con una tendencia a circular de una manera más arriesgada. Caso del uso de los airbag en USA y en Alemania. El fenómeno ha sido acuñado en el mundo científico como «la Teoría del riesgo constante».

2. Las carreteras por las que circulamos. Los diseños, los tipos de carreteras, los nuevos sistemas de seguridad, la generalización de atenuadores de impacto, etc., van a sufrir cambios significativos en pocos años. 
De todos ellos, en los que hacen referencia al complejo mundo de la información al conductor y la señalización, la psicología y los especialistas en temas perceptivos podrían aportar datos valiosos para el diseño, sobre todo, teniendo en cuenta el progresivo envejecimiento de la población.

3. La investigación de los accidentes y las causas que los motivaron, es preciso conocer con exactitud todas las circunstancias en las que se producen los accidentes las causas que los desencadenaron, los grupos de riesgo a los que afectan, etc., toda vez que esto constituye parte de la actividad preventiva.

Asimismo, se debe superar el modelo actual de recogida de datos de los accidentes, que en buena medida está enfocado a aspectos legales y de responsabilidad jurídica más que al conocimiento en profundidad de los motivos que desencadenaron los siniestros.

4. Sanciones, es evidente que el estricto cumplimiento de las normas es lo único que nos puede hacer prever cuál va a ser el comportamiento de los demás, cuando compartimos el espacio común de las carreteras. Sobre el punto la psicología del tráfico y la seguridad deberá aportar en el futuro: sofisticación de la tecnología de detección de los infractores; mejorar la eficacia de las sanciones y, lógicamente, potenciar la formación del conductor.

5. Formación de los conductores, a partir de los años 50 las investigaciones empezaron a mostrar la importancia del factor humano y en concreto de la buena formación integral del conductor, como un elemento clave para evitar la accidentalidad y mejorar la seguridad vial.

Habría que reforzar y dirigir una buena parte de la enseñanza de los conductores, hacia los factores de riesgo que desencadenan fallas humanas y el cambio de actitudes, dimensiones actualmente bastante olvidada en algunos países, especialmente en el nuestro, pese a que todos los estudios coinciden en que el error humano está implicado entre el 70 y $90 \%$ de los accidentes.

Otro aspecto es el referente a la conducción defensiva y los primeros auxilios. Las investigaciones nos dicen que el $40 \%$ de las víctimas de los accidentes de tránsito mueren en los primeros 25 minutos, y una buena parte de los heridos quedan con secuelas permanentes, en muchos casos porque los conductores no saben qué hacer desde que se produce un accidente, hasta que llegan los servicios asistenciales.

6. Otros grupos humanos: niños, peatones, ciclistas, jóvenes y personas mayores de 65 años, en el caso del grupo de los niños por ejemplo, según las estadísticas, en los países desarrollados las enfermedades representan el 30\% de las muertes de los niños entre los 1-16 años. Les siguen en importancia los accidentes de tránsito, que ocasionan más del $20 \%$ de las muertes en este grupo de edad. El desarrollar investigaciones que sitúen espaciotemporalmente los siniestros y conocer todos los problemas sensoriales, perceptivos, atencionales, etc., que les hace más proclive a los accidentes, son, sin duda, la base más sólida para desarrollar estrategias psicosociales de tipo preventivo.

7. Factores clásicos de riesgo en la conducción, se encuentran el alcohol, la velocidad, las drogas y los fármacos y el grupo formado por el sueño, la fatiga y las distracciones. 


\section{Hipótesis}

\section{Hipótesis general}

Los conductores de servicio público (taxistas, microbuseros y choferes interprovinciales) y los automovilistas particulares de Lima Metropolitana presentan patrones de conducta diferenciados en contextos de tráfico.

\section{Hipótesis específicas}

$\mathrm{H}_{1}$ Existen diferencias significativas en los patrones de conducta en contextos de tráfico entre los automovilistas particulares y los microbuseros de Lima Metropolitana.

$\mathrm{H}_{2}$ Existen diferencias significativas en los estilos de conducta en contextos de tráfico entre los automovilistas particulares y los taxistas de Lima Metropolitana.

$\mathrm{H}_{3} \quad$ Existen diferencias significativas en los patrones de conducta en contextos de tráfico entre los conductores particulares y los choferes interprovinciales.

$\mathrm{H}_{4} \quad$ Existen diferencias significativas en los estilos de comportamiento en contextos de tráfico entre los microbuseros y los taxistas de Lima Metropolitana.

$\mathrm{H}_{5}$ Existen diferencias significativas en los patrones de comportamiento en contextos de tráfico entre los microbuseros y los choferes de ómnibus interprovinciales.

$\mathrm{H}_{6} \quad$ Existen diferencias significativas en los estilos de conducta en contextos de tráfico entre los taxistas y los choferes de ómnibus interprovinciales.

\section{MÉTODOS}

\section{Diseño de Investigación}

El diseño de investigación corresponde al descriptivo comparativo que parte de la consideración de dos o más investigaciones descriptivas simples; esto es, recolectar información relevante en varias muestras con respecto a un mismo fenómeno o aspecto de interés y luego caracterizar este fenómeno en base a la comparación de los datos recogidos, pudiendo hacerse esta comparación en los datos generales o en una categoría de ellos. Por ejemplo, queremos dar una descripción general de la situación actual, que tenga aceptación de consenso de los pueblos jóvenes.

\section{Universo y muestra de investigación}

La población estuvo conformada por los choferes de servicio público (microbuseros, taxistas y choferes interprovinciales) y particulares que conducen vehiculos en Lima Metropolitana. Para el presente estudio se ha trabajado con una muestra representativa de 740 casos, la cual fue seleccionada a través de un muestreo no-probabilístico de tipo intencional o intencionado. En este tipo de muestreo quien selecciona la muestra lo que busca es que ésta sea representativa de la población donde es extraída. Lo importante es que dicha 
representatividad se da en base a una opinión o intención particular de quien selecciona la muestra y, por lo tanto, la evaluación de la representatividad es subjetiva.

\section{Composición de la muestra}

En líneas generales, la muestra ha estado conformada por 740 choferes varones que conducen vehículos en Lima Metropolitana cuyas edades han oscilado entre los 19 y los 69 años. De ese total, 249 son automovilistas particulares y 491 son conductores de servicio público, entre taxistas (152); microbuseros (228); y choferes interprovinciales (111), respectivamente.

Las características de la muestra se detallan entre las tablas del 1 al 11.

Tabla 1. Composición de la muestra por brevete o tipo de licencia deconducir

\begin{tabular}{lcc}
\hline Brevete & Frecuencia & Porcentaje (\%) \\
\hline Particular & 362 & 48,9 \\
Profesional & 378 & 51,1 \\
\hline Total & 740 & 100,0 \\
\hline
\end{tabular}

En la tabla 1 podemos apreciar que la muestra ha estado conformada por 378 choferes con licencia profesional $(51,1 \%)$, y 362 conductores con brevete particular $(48,9 \%)$, lo cual infiere un equilibrio en cuanto al número de sujetos de ambos grupos.

Tabla 2. Composición de la muestra por tipo de automóvil que conduce.

\begin{tabular}{lcc}
\hline Automóvil & Frecuencia & Porcentaje \\
\hline Particular & 249 & 33,6 \\
Taxi & 152 & 20,5 \\
Ómnibus urbano & 228 & 30,8 \\
Ómnibus interprovincial & 111 & 15,0 \\
\hline Total & 740 & 100,0 \\
\hline
\end{tabular}

En la tabla 2 observamos que la mayor proporción de sujetos examinados ha sido de conductores particulares $(33,6 \%)$, así como de choferes de microbuses u ómnibus urbanos $(30,8 \%)$, el grupo de automovilistas de ómnibus interprovinciales ha sido de 111 , que corresponde a la menor proporción (15\%). El grupo de taxistas tiene un porcentaje de $20 \%$, que corresponde a 152 sujetos examinados. 
Tabla 3. Composición de la muestra por edad cronológica.

\begin{tabular}{|c|c|c|c|c|c|}
\hline Edad & Frecuencia & Porcentaje & Edad & Frecuencia & Porcentaje \\
\hline 19 & 4 & ,5 & 45 & 38 & 5,1 \\
\hline 20 & 4 &, 5 & 46 & 13 & 1,8 \\
\hline 21 & 6 &, 8 & 47 & 14 & 1,9 \\
\hline 22 & 5 &, 7 & 48 & 27 & 3,6 \\
\hline 23 & 2 &, 3 & 49 & 22 & 3,0 \\
\hline 24 & 13 & 1,8 & 50 & 23 & 3,1 \\
\hline 25 & 12 & 1,6 & 51 & 18 & 2,4 \\
\hline 26 & 21 & 2,8 & 52 & 18 & 2,4 \\
\hline 27 & 17 & 2,3 & 53 & 11 & 1,5 \\
\hline 28 & 27 & 3,6 & 54 & 14 & 1,9 \\
\hline 29 & 12 & 1,6 & 55 & 11 & 1,5 \\
\hline 30 & 27 & 3,6 & 56 & 7 &, 9 \\
\hline 31 & 19 & 2,6 & 57 & 9 & 1,2 \\
\hline 32 & 24 & 3,2 & 58 & 11 & 1,5 \\
\hline 33 & 21 & 2,8 & 59 & 5 &, 7 \\
\hline 34 & 15 & 2,0 & 60 & 8 & 1,1 \\
\hline 35 & 36 & 4,9 & 61 & 5 &, 7 \\
\hline 36 & 22 & 3,0 & 62 & 4 &, 5 \\
\hline 37 & 11 & 1,5 & 63 & 4 &, 5 \\
\hline 38 & 29 & 3,9 & 64 & 4 &, 5 \\
\hline 39 & 26 & 3,5 & 65 & 3 &, 4 \\
\hline 40 & 30 & 4,1 & 66 & 1 &, 1 \\
\hline 41 & 18 & 2,4 & 68 & 3 &, 4 \\
\hline 42 & 22 & 3,0 & 69 & 1 &, 1 \\
\hline 43 & 22 & 3,0 & No & 5 &, 7 \\
\hline \multirow[t]{2}{*}{44} & 16 & 2,2 & Contestaron & & \\
\hline & & & Total & 740 & 100,0 \\
\hline
\end{tabular}

En la tabla 3, podemos observar que la muestra ha oscilado entre los 19 y 69 años, apreciándose un grupo bastante heterogéneo. 
Tabla 4. Composición de la muestra estado civil.

\begin{tabular}{lll}
\hline Estado Civil & Frecuencia & Porcentaje \\
\hline Soltero & 161 & 21,8 \\
Casado & 356 & 48,1 \\
Viudo & 11 & 1,5 \\
Divorciado & 36 & 4,9 \\
Conviviente & 176 & 23,8 \\
\hline Total & 740 & 100,0 \\
\hline
\end{tabular}

En la tabla 4, observamos que el mayor porcentaje de choferes examinados son casados $(48,1 \%)$; a este grupo le sigue un grupo de conductores que son convivientes $(23,8 \%)$; luego están los choferes solteros en una proporción de $21,8 \%$ y luego los divorciados y viudos en un porcentaje de $4,9 \%$ y $1,5 \%$, respectivamente.

Tabla 5. Composición de la muestra por grado de instrucción.

\begin{tabular}{lll}
\hline Grado de instrucción & Frecuencia & Porcentaje \\
\hline Primaria & 37 & 5,0 \\
Secundaria & 354 & 47,8 \\
Superior & 266 & 35,9 \\
Técnica & 80 & 10,8 \\
No contestaron & 3 &, 4 \\
\hline Total & 740 & 100,0 \\
\hline
\end{tabular}

En la tabla 5, apreciamos que la mayoría de los choferes examinados nos refieren que tienen educación secundaria (47,8\%) y superior (35,9\%). Ambos grupos corresponden a más del $80 \%$ de la muestra. Existe un 10,8\% con educación técnica y un $5 \%$ con primaria.

Tabla 6. Composición de la muestra por lugar de nacimiento.

\begin{tabular}{lll}
\hline & Frecuencia & Porcentaje \\
\hline Lima & 349 & 47,2 \\
Provincia & 389 & 52,6 \\
No contestaron & 2 &, 3 \\
\hline Total & 740 & 100,0 \\
\hline
\end{tabular}


En la tabla 6, apreciamos que un poco más de la mitad de conductores examinados han nacido en provincias $(52,6 \%)$, en tanto que el $47,2 \%$ han nacido en Lima.

Tabla 7. Composición de la muestra por pertenencia del vehículo.

\begin{tabular}{lll}
\hline Pertenencia del vehículo & Frecuencia & Porcentaje \\
\hline Propio & 350 & 47,3 \\
Alquilado & 227 & 30,7 \\
Empresa & 163 & 22,0 \\
\hline Total & 740 & 100,0 \\
\hline
\end{tabular}

En la tabla 7 se aprecia que la mayor proporción de la muestra estudiada $(47,3 \%)$, es propietario de su vehículo, en tanto que el $30,7 \%$ maneja vehículo alquilado y el $22 \%$ opera vehículo perteneciente a la Empresa donde labora.

Tabla 8. Composición de la muestra por accidentes de tránsito.

\begin{tabular}{lll}
\hline Ha tenido accidentes de tránsito & Frecuencia & Porcentaje \\
\hline Nunca & 277 & 37,4 \\
$\mathbf{1}$ vez & 268 & 36,2 \\
$\mathbf{2}$ veces & 122 & 16,5 \\
3 o más veces & 71 & 9,6 \\
No contestaron & 2 &, 3 \\
\hline Total & 740 & 100,0 \\
\hline
\end{tabular}

En la tabla 8 , apreciamos que las mayores proporciones se encuentran entre los que nunca han tenido accidentes $(37,4 \%)$, y los que lo han tenido una vez $(36,2)$. Ambos grupos cubren más del $70 \%$ de la muestra.

Tabla 9. Composición de la muestra por problemas de salud.

\begin{tabular}{lll}
\hline Problema de salud & Frecuencia & Porcentaje \\
\hline No & 603 & 81,5 \\
Sí & 137 & 18,5 \\
\hline Total & 740 & 100,0 \\
\hline
\end{tabular}

De acuerdo a lo observado en la tabla 9 , un $81,5 \%$ de la muestra de conductores manifiesta no tener problemas de salud y un $18,5 \%$, es decir, 137 choferes refieren tener problemas de salud. 
Tabla 10. Composición de la muestra por problemas familiares.

\begin{tabular}{lll}
\hline Problema Familiar & Frecuencia & Porcentaje \\
\hline No & 634 & 85,7 \\
Sí & 106 & 14,3 \\
\hline Total & 740 & 100,0 \\
\hline
\end{tabular}

En la tabla 10, podemos apreciar que un $14,3 \%$ de la muestra (106), manifiestan tener problemas familiares, en tanto que un $85,7 \%$ no refiere presentarlos.

Tabla 11. Composición de la muestra por problemas emocionales.

\begin{tabular}{lll}
\hline Problema emocional & Frecuencia & Porcentaje \\
\hline No & 679 & 91,8 \\
Sí & 61 & 8,2 \\
\hline Total & 740 & 100,0 \\
\hline
\end{tabular}

En la tabla 11, observamos que un $8,2 \%$ de los conductores (61) refieren presentar problemas emocionales, en tanto que un $91,8 \%$ manifiesta no presentarlos.

\section{Variables del estudio}

\section{Variable dependiente}

- $\quad$ Patrones de conducta en contextos de tráfico.

\section{Variables independientes asignadas}

- Edad cronológica

- $\quad$ Estado civil

- Grado de instrucción

- Lugar de nacimiento

- $\quad$ Tipo de licencia de conducir

- Pertenencia del vehículo

- Dedicación a la conducción

- Frecuencia de accidentes de tránsito

- Problemas de salud

- Problemas emocionales 


\section{Técnicas e Instrumentos de recolección de datos}

\section{Cuestionario de Personalidad PSS}

\section{Ficha técnica}

En el presente estudio se ha utilizado el cuestionario de personalidad PSS, el cual forma parte de la $\mathrm{BC}$, batería de conductores que procede del departamento $\mathrm{I}+\mathrm{D}$ de TEA Ediciones y J.L. Fernández-Seara.

La administración puede ser individual y colectiva y demora aproximadamente 15 minutos; y en cuanto a la significación el instrumento examina patrones de conducta de los automovilistas en contextos de tráfico y la tipificación nos ofrece baremos en centiles.

\section{Descripción de la escala de personalidad}

Esta escala ha sido diseñada, en primer lugar, para analizar las características comportamentales del sujeto en contextos de tráfico y, en segundo lugar, para ver qué tipo de conductas elicitan las situaciones de tráfico. En definitiva, se trata de determinar la existencia de ciertos patrones de conducta o estilos de comportamiento.

\section{Marco Teórico}

Los supuestos teóricos sobre los que se apoya esta escala son, por una parte, la existencia de ciertos patrones o estilos de comportamiento estables y permanentes a lo largo del tiempo y de las situaciones (estabilidad y consistencia transituacional) y, por otra, el reconocimiento del peso de las situaciones en la especificación de la conducta (Mischel, 1968; Bowers, 1973). Se ha intentado analizar, pues, el peso de la generalidad intrapsíquica vs. la especificidad situacional en contextos concretos de la conducción.

\section{Estructura de la escala}

Esta escala está estructurada en dos partes:

- Parte primera: Rasgos situacionales. En ella se describen características comportamentales de los sujetos (ítems), y las circunstancias relacionadas con el tráfico, en que se ponen de manifiesto.

- Parte segunda: Situacional. Se categorizan situaciones de tráfico y alternativas de comportamiento que se pueden especificar en ellas.

En la primera se estudia la relación rasgos-situaciones en el contexto de tráfico, y en la segunda, el papel de las situaciones de tráfico en la conducta del individuo al volante.

\section{Tipología}

A partir de la información recabada de las dos partes de la escala se pueden obtener dos patrones de conducta o estilos de comportamiento denominados Tipo S y Tipo N, que se refieren a dos patrones de personalidad o estilos de conducta a la hora de realizar la conducción en automóvil. 
Patrón de conducta Tipo S. A nivel descriptivo podemos decir que los sujetos de patrón de conducta tipo S:

- Conducen bajo una percepción y aceptación del riesgo adecuada.

- Realizan una conducción precavida, atenta y responsable.

- Conducen con un alto nivel de seguridad.

- Controlan adecuadamente la conducta.

En resumen, son seguros, prudentes, responsables, atentos y precavidos (tipo ideal).

Patrón de conducta Tipo $\mathbf{N}$.

- Presentan una deficiente percepción del riesgo.

- Son incapaces de reaccionar adecuadamente al «estrés emocional».

- Manifiestan impulsividad, impaciencia, agresividad y tendencias antisociales.

- $\quad$ Poseen escaso control personal.

- Muestran conductas de falta de respeto a los demás usuarios y a las normas de tráfico.

- Tienden a exteriorizar sus respuestas a la frustración de forma excesiva y poco adecuada. La menor contrariedad -atascos, retenciones, cruces indebidos-, se traduce en irritabilidad y accesos de cólera desproporcionados.

- En síntesis, su conducta se manifiesta desajustada, de alto riesgo y peligrosa.

\section{Material para la aplicación}

Comprende un cuadernillo de preguntas compuesto por 57 ítemes; una hoja de respuestas o protocolo y una plantilla de corrección.

\section{Normas de corrección, puntuación e interpretación}

En la corrección de la prueba de personalidad PSS, es necesario obtener las puntuaciones S (primera parte), $\mathrm{N}$ (segunda parte) y $\mathrm{T}$ (escala total); la tercera es el resultado de sumar las dos primeras. La tarea se facilita con el uso de la plantilla de corrección.

Para su interpretación global, se analiza la puntuación centil de la Escala total, distinguiendo si el valor centil es superior a 65. Entonces se definirá al sujeto con las características dadas al Tipo S. Por el contrario, si la puntuación centil es baja (inferior a 40), se le aplicarán las características del Tipo $\mathbf{N}$.

La ubicación en la escala centil nos permitirá descubrir su «estilo o patrón de conducta» y predecir, en cierta manera, su comportamiento futuro como conductor, sobre todo en el sentido de poder discriminar el umbral subjetivo de riesgo en la conducción.

\section{Encuesta de datos personales}

Complementariamente al instrumento utilizado, se ha aplicado una pequeña encuesta de 18 preguntas que constituye el registro de variables independientes asignadas, en donde se ha 
detectado información referente a: edad cronológica; estado civil; grado de instrucción; lugar de nacimiento; tipo de licencia de conducir; pertenencia del vehículo; dedicación a l conducción; frecuencia de accidentes de tránsito; problemas de salud; problemas visuales; problemas auditivos; problemas de motricidad; problemas familiares; problemas emocionales, entre otras.

\section{RESULTADOS}

A continuación de exponen los principales resultados alcanzados:

Tabla 12. Análisis de la confiabilidad de toda la prueba $\mathrm{S}+\mathrm{N}$.

\begin{tabular}{|c|c|c|c|c|c|c|c|}
\hline Ítemes & Media & D. E. & rit & Ítemes & Media & D. E. & rit \\
\hline cpc1 & 0,83 & 1,16 & 0,28 & cpc30 & $-0,17$ & 1,08 & 0,33 \\
\hline срc2 & 1,08 & 0,75 & 0,21 & срc31 & 0,14 & 1,25 & 0,40 \\
\hline срс3 & $-1,84$ & 1,21 & 0,11 & срс32 & 0,37 & 0,95 & 0,46 \\
\hline cpc4 & 0,36 & 1,02 & 0,43 & cpc33 & 0,84 & 0,93 & 0,14 \\
\hline сpc5 & $-1,42$ & 0,87 & 0,10 & срc34 & 0,26 & 1,01 & 0,41 \\
\hline срс6 & $-0,78$ & 1,60 & 0,02 & cpc35 & $-0,09$ & 0,99 & 0,48 \\
\hline cpc7 & $-0,15$ & 1,70 & 0,29 & cpc36 & $-0,33$ & 0,96 & 0,32 \\
\hline срс8 & $-1,45$ & 0,90 & 0,05 & cpc37 & 0,20 & 0,91 & 0,41 \\
\hline срс9 & $-0,61$ & 0,78 & 0,27 & cpc38 & 0,18 & 0,89 & 0,28 \\
\hline cpc10 & $-1,08$ & 0,98 & 0,25 & срс39 & $-0,08$ & 0,76 & 0,44 \\
\hline cpc11 & $-0,62$ & 0,62 & 0,23 & срc40 & 0,24 & 0,86 & 0,30 \\
\hline срс12 & 0,73 & 1,16 & 0,40 & срс41 & $-0,60$ & 0,90 & 0,10 \\
\hline cpc13 & $-0,26$ & 1,06 & 0,24 & срс42 & 0,01 & 0,99 & 0,45 \\
\hline cpc14 & 1,99 & 1,20 & 0,27 & срс43 & $-0,41$ & 1,06 & 0,44 \\
\hline cpc15 & $-1,13$ & 1,16 & 0,33 & cpe44 & $-0,64$ & 0,99 & 0,08 \\
\hline срc16 & $-0,47$ & 0,62 & 0,17 & cpc45 & 0,65 & 0,80 & 0,18 \\
\hline cpc17 & 1,99 & 1,14 & 0,31 & сpc46 & $-0,16$ & 1,01 & 0,46 \\
\hline cpc18 & 1,46 & 1,00 & 0,13 & срc47 & $-0,18$ & 0,85 & 0,14 \\
\hline сpc19 & $-1,04$ & 1,14 & 0,44 & срс48 & $-0,02$ & 0,92 & 0,46 \\
\hline срс20 & $-0,33$ & 1,25 & 0,17 & срс49 & 0,26 & 1,15 & 0,34 \\
\hline сpc21 & $-0,04$ & 1,18 & 0,30 & $\operatorname{cpc50}$ & 0,08 & 1,28 & 0,40 \\
\hline срс22 & 0,16 & 1,30 & 0,48 & cpc51 & $-0,16$ & 0,85 & 0,25 \\
\hline срс23 & $-0,08$ & 1,27 & 0,51 & cpc52 & 0,06 & 1,07 & 0,27 \\
\hline срc24 & $-0,06$ & 0,92 & 0,28 & cpc53 & $-0,29$ & 1,07 & 0,26 \\
\hline cpc25 & $-1,00$ & 1,04 & 0,43 & cpc54 & 0,29 & 0,89 & 0,26 \\
\hline срс26 & $-0,21$ & 1,34 & 0,36 & cpc55 & $-0,08$ & 0,96 & 0,39 \\
\hline сpc27 & $-0,38$ & 1,21 & 0,31 & cpc56 & 0,33 & 0,78 & 0,39 \\
\hline срc28 & 0,28 & 0,98 & 0,34 & cpc57 & 1,64 & 1,63 & 0,28 \\
\hline Cpc29 & 0,49 & 0,21 & 0,27 & & & & \\
\hline \multicolumn{8}{|c|}{ Alfa de Cronbach $=0,86$} \\
\hline
\end{tabular}


Los resultados obtenidos en la tabla 12 , revelan un Alfa de Cronbach de 0,86 que nos indica la existencia de puntajes confiables a nivel global del Cuestionario PSS.

Debemos indicar que los estilos de comportamiento en contextos de tráfico que mide la prueba es consecuencia del puntaje total $\mathrm{S}+\mathrm{N}$ y cuyos valores pueden resultar positivos o negativos.

\section{Comparaciones}

Tabla 13. Análisis comparativo de las variables estudiadas por vehículo particular o de servicio público que se conduce.

\begin{tabular}{llllll}
\hline \multirow{2}{*}{ Variables } & \multicolumn{3}{c}{$\begin{array}{l}\text { Particular } \\
\mathbf{N}=\mathbf{3 6 2}\end{array}$} & \multicolumn{4}{c}{$\begin{array}{l}\text { Servicio público } \\
\mathbf{N}=\mathbf{3 7 8}\end{array}$} & $\mathbf{Z}$ \\
\cline { 2 - 5 } & $\mathbf{M}$ & D. E. & $\mathbf{M}$ & D. E. & \\
\hline $\begin{array}{l}\text { PSS - 1 } \\
\text { (Factor S) }\end{array}$ & $-2,99$ & 6,11 & $-2,47$ & 5,43 & $-1,23$ \\
$\begin{array}{l}\text { PSS - 2 } \\
\text { (Factor N) }\end{array}$ & $-0,85$ & 14,95 & 3,80 & 15,30 & $-4,17^{* * *}$ \\
PSS To tal & $-3,84$ & 18,60 & 1,33 & 18,05 & $-3,83^{* * *}$ \\
\hline
\end{tabular}

$* * * \mathrm{p}<, 001$

En la tabla 13, podemos apreciar, en primer lugar, que no existen diferencias significativas en el factor S, referidas al factor de la estabilidad y consistencia transituacional y, por lo tanto, a la existencia de patrones de comportamiento estables y permanentes a lo largo del tiempo entre los conductores particulares y de servicio público. Más bien, ambos grupos presentan valores promedio negativos. En segundo lugar, observamos la existencia de diferencias significativas en el factor $\mathrm{N}$, referido al papel de las situaciones de tráfico en la conducta del individuo al volante, entre los choferes particulares y de servicio público, a favor del grupo de conductores de servicio público. Finalmente, existen diferencias estadísticamente significativas a nivel global, en cuanto a estilos de comportamiento, en contextos de tráfico, entre los choferes particulares y los de servicio público, a favor de los choferes del segundo grupo. 
Tabla 14. Análisis de varianza de un factor de las variables estudiadas por tipo de vehículo específico de servicio público o particular que se conduce.

\begin{tabular}{llllll}
\hline Variables & $\begin{array}{l}\text { Particular } \\
\mathbf{N = 2 4 9}\end{array}$ & $\begin{array}{l}\text { Taxi } \\
\mathbf{N = 1 5 2}\end{array}$ & $\begin{array}{l}\text { Ómnibus } \\
\text { urbano } \\
\mathbf{N}=\mathbf{2 2 8}\end{array}$ & $\begin{array}{l}\text { Ómnibus } \\
\text { interprovincial } \\
\mathbf{N}=\mathbf{1 1 1}\end{array}$ & \multirow{2}{*}{$\mathbf{~}$} \\
\cline { 2 - 5 } & $\mathbf{M}$ & $\mathbf{M}$ & $\mathbf{M}$ & $\mathbf{M}$ & \\
\hline $\begin{array}{l}\text { PSS - 1 } \\
\text { (Factor S) }\end{array}$ & $-2,23 \mathrm{a}$ & $-3,63 \mathrm{ab}$ & $-3,12 \mathrm{c}$ & $-1,78 \mathrm{bc}$ & $3,23 *$ \\
$\begin{array}{l}\text { PSS } \mathbf{2} \\
\text { (Factor N) }\end{array}$ & $0,65 \mathrm{a}$ & $0,43 \mathrm{~b}$ & $0,77 \mathrm{c}$ & $6,55 \mathrm{abc}$ & $4,78 * *$ \\
PSS Total & $-1,58 \mathrm{a}$ & $-3,20 \mathrm{~b}$ & $-2,36 \mathrm{c}$ & $4,77 \mathrm{abc}$ & $4,85 * *$ \\
\hline
\end{tabular}

$* \mathrm{p}<.05 * * \mathrm{p}<.01$

Diferencias significativas por pares de medias a través del test de Scheffe: a, b, c

En la tabla 14, podemos observar, en primer término, diferencias significativas en el factor $\mathrm{S}$, entre choferes particulares y taxistas, a favor de los particulares; entre taxistas y choferes interprovinciales, a favor de los interprovinciales; y entre microbuseros y conductores de ómnibus interprovinciales, a favor de los choferes interprovinciales. En este caso, se aprecia que todos los promedios presentan valores negativos.

En segundo término, podemos apreciar diferencias significativas en el factor $\mathrm{N}$, entre choferes particulares y conductores de ómnibus interprovinciales, a favor de los segundos; entre taxistas y choferes de ómnibus interprovinciales, a favor del segundo grupo; $y$, entre microbuseros y choferes de ómnibus interprovinciales, a favor de los conductores interprovinciales. Asimismo, todos las medias o promedios presentan valores positivos.

En cuanto a los estilos de comportamiento globales, en contextos de tráfico, se aprecian diferencias significativas entre choferes particulares y los que conducen ómnibus interprovinciales, a favor de los segundos; entre taxistas y choferes interprovinciales, a favor del segundo grupo; y entre microbuseros y choferes de ómnibus interprovinciales, a favor del segundo grupo. En suma, los choferes de ómnibus interprovinciales presentan comparativamente estilos de comportamiento en contextos de tráfico más adecuados y positivos, frente a los choferes particulares, microbuseros y taxistas. Estos últimos inclusive, presentan valores negativos. 
Tabla 15. Análisis de varianza de un factor por pertenencia del vehículo.

\begin{tabular}{|c|c|c|c|c|}
\hline \multirow{2}{*}{ Variables } & $\begin{array}{l}\text { Propio } \\
\mathbf{N}=\mathbf{3 5 0}\end{array}$ & $\begin{array}{l}\text { Milado } \\
\mathbf{N}=\mathbf{2 2 7}\end{array}$ & $\begin{array}{l}\text { Fipresa } \\
N=16\end{array}$ & \multirow{2}{*}{$\mathbf{F}$} \\
\hline & $\mathbf{M}$ & $\mathbf{M}$ & $\mathbf{M}$ & \\
\hline $\begin{array}{l}\text { PSS - } 1 \\
\text { (Factor S) }\end{array}$ & $-2,73$ & $-3,26$ & $-1,98$ & 2,34 \\
\hline $\begin{array}{lr}\text { PSS } & -2 \\
(\text { Factor } & \text { N) }\end{array}$ & $0,29 \mathrm{a}$ & $-0,01 \mathrm{~b}$ & $6,32 \mathrm{ab}$ & $10,56 * * *$ \\
\hline PSS Total & $-2,44 \mathrm{a}$ & $-3,26 b$ & $4,34 \mathrm{ab}$ & $9,75 * * *$ \\
\hline
\end{tabular}

$* * * \mathrm{p}<, 001$

\section{Diferencias significativas por pares de medias a través del test de Scheffe: a, b}

En la tabla 15, a nivel global, encontramos diferencias significativas en los patrones de comportamiento en contextos de tráfico en relación a la pertenencia del vehículo, entre los que manejan su vehículo propio y los que conducen vehículos de una empresa, a favor de los segundos. Asimismo, hallamos diferencias significativas entre los que conducen vehículos alquilados respecto de los que manejan vehículos de empresas, a favor también de los segundos.

Tabla 16 Análisis de varianza de un factor por tiempo en la conducción.

\begin{tabular}{|c|c|c|c|c|c|c|}
\hline \multirow{2}{*}{ Variables } & $\begin{array}{l}1-5 \text { años } \\
\mathrm{N}=\mathbf{2 1 1} \\
\end{array}$ & $\begin{array}{l}\text { 6-10 años } \\
N=200\end{array}$ & $\begin{array}{l}11-15 \text { años } \\
N=111\end{array}$ & $\begin{array}{l}\text { 16-20 años } \\
N=85\end{array}$ & $\begin{array}{l}21 \text { a más } \\
N=133\end{array}$ & \multirow{2}{*}{$\mathbf{F}$} \\
\hline & $\mathbf{M}$ & M & M & M & $\mathbf{M}$ & \\
\hline $\begin{array}{l}\text { PSS - } 1 \\
\text { (Factor S) }\end{array}$ & $-3,28 \mathrm{a}$ & $-2,95 \mathrm{~b}$ & $-2,90 \mathrm{c}$ & $-2,69$ & $-1,38 a b c$ & $2,40 *$ \\
\hline $\begin{array}{l}\text { PSS - } 2 \\
\text { (Factor N) }\end{array}$ & $-0,89 a b$ & $-0,19 \mathrm{c}$ & $1,44 d$ & $3,58 \mathrm{a}$ & $6,70 \mathrm{bcd}$ & $6,31 * * *$ \\
\hline PSS Total & $-4,17 a b$ & $-3,14 c$ & $-1,46 \mathrm{~d}$ & $0,88 \mathrm{a}$ & $5,32 \mathrm{bcd}$ & $6,50 * * *$ \\
\hline
\end{tabular}

$* \mathrm{p}<, 05 * * \mathrm{p}<.01 * * * \mathrm{p}<, 001$ 


\section{Diferencias significativas por pares de medias a través del test de Scheffe: a,b,c,d}

En la tabla 16, a nivel global encontramos diferencias significativas en los estilos de comportamiento en relación a tiempo en la conducción entre los que conducen entre 1-5 años, respecto de los que conducen entre los 16-20 años, a favor de los segundos; asimismo, se hallan diferencias entre los que manejan entre los 1-5, respecto de los que conducen entre los 21 o más años; del mismo modo encontramos diferencias entre los choferes que manejan entre los 6-10 años, respecto de los que lo hacen entre los 21 o más años; finalmente, encontramos diferencias entre los que conducen entre los 11-15 años, respecto de los que manejan entre los 21 o más años. En suma, a mayor tiempo en la conducción van mejorando gradualmente los estilos de comportamiento en contextos de tráfico.

Tabla 17. Análisis de varianza de un factor por accidentes de tránsito.

\begin{tabular}{llllll}
\hline \multirow{2}{*}{ Variables } & $\begin{array}{l}\text { Nunca } \\
\mathbf{N = 2 7 7}\end{array}$ & $\begin{array}{l}\mathbf{1} \text { vez } \\
\mathbf{N = 2 8}\end{array}$ & $\begin{array}{l}\mathbf{2} \text { veces } \\
\mathbf{N}=\mathbf{1 2 2}\end{array}$ & $\begin{array}{l}\mathbf{3} \text { o má veces } \\
\mathbf{N}=\mathbf{7 1}\end{array}$ & \multirow{2}{*}{$\mathbf{F}$} \\
\cline { 2 - 5 } & $\mathbf{M}$ & $\mathbf{M}$ & $\mathbf{M}$ & $\mathbf{M}$ & \\
\hline $\begin{array}{l}\text { PSS - 1 } \\
\text { (Factor S) }\end{array}$ & $-1,10 \mathrm{abc}$ & $-3,05 \mathrm{ad}$ & $-4,06 \mathrm{~b}$ & $-5,42 \mathrm{~cd}$ & $15,84 * * *$ \\
$\begin{array}{l}\text { PSS - 2 } \\
\text { (Factor } \mathbf{N})\end{array}$ & $3,54 \mathrm{ab}$ & $2,41 \mathrm{c}$ & $-0,56 \mathrm{ad}$ & $-5,97 \mathrm{bcd}$ & $8,64 * * *$ \\
PSS Total & $2,43 \mathrm{abc}$ & $-0,64 \mathrm{ade}$ & $-4,61 \mathrm{bdf}$ & $-11,39 \mathrm{cef}$ & $12,90 * * *$ \\
\hline
\end{tabular}

$* * * \mathrm{p}<, 001$

Diferencias significativas por pares de medias a través del test de Scheffe: a,b,c,d,e

En la tabla 17 se aprecian a nivel global diferencias significativas en los estilos de comportamiento en relación a los accidentes de tránsito, entre los que nunca han tenido accidentes respecto de los que han presentado 1 accidente; entre los que nunca han tenido accidentes respecto de los que han tenido dos accidentes; entre los que nunca han tenido accidentes respecto de los que han tenido tres o más accidentes; entre los que han tenido 1 vez respecto de los que han presentado dos accidentes; entre los que han tenido una vez respecto de los que han presentado tres o más accidentes y finalmente entre los que han presentado dos respecto de los que han tenido tres o más accidentes. En suma, la frecuencia de accidentes de tránsito nos revela estilos de comportamiento negativos e inadecuados en contextos de tráfico, frente a la ausencia de accidentes que se expresa en valores positivos o si se quiere en una buena conducción. 
Tabla 18. Análisis comparativo de las variables estudiadas por problema de salud.

\begin{tabular}{|c|c|c|c|c|c|}
\hline \multirow{2}{*}{ Variables } & \multicolumn{2}{|c|}{$\begin{array}{l}\text { No } \\
N=603 \\
\end{array}$} & \multicolumn{2}{|c|}{$\begin{array}{l}\text { Sí } \\
\mathbf{N}=137\end{array}$} & \multirow{2}{*}{$\mathbf{Z}$} \\
\hline & Media & D. E. & $\mathbf{M}$ & D. E. & \\
\hline $\begin{array}{l}\text { PSS - 1 } \\
\text { (Factor S) }\end{array}$ & $-2,49$ & 5,74 & $-3,75$ & 5,86 & $2,31 *$ \\
\hline $\begin{array}{l}\text { PSS - } 2 \\
(\text { Factor N) }\end{array}$ & 1,87 & 14,92 & 0,00 & 16,84 & 1,29 \\
\hline PSS Total & $-0,62$ & 18,01 & $-3,75$ & 20,36 & 1,20 \\
\hline
\end{tabular}

En la tabla 18, podemos apreciar la existencia de difrencias estadísticamente significativas en el factor $S$ entre los choferes que manifiestan problemas de salud, respecto de los que no los presentan, esto es, a favor de los segundos, lo cual estaría indicando que la buena salud guarda una relación positiva con el estilo $S$ de conducción positiva. Sin embargo, no se aprecian diferencias significativas en el factor $\mathrm{N}$ y en los estilos globales de comportamiento en contextos de tráfico en la variable problemas de salud.

Tabla 19. Análisis comparativo de las variables estudiadas por problemas familiares.

\begin{tabular}{|c|c|c|c|c|c|}
\hline \multirow{2}{*}{ Variable } & \multicolumn{2}{|c|}{$\begin{array}{l}\text { No } \\
N=634\end{array}$} & \multicolumn{2}{|c|}{$\begin{array}{l}\text { Sí } \\
N=106\end{array}$} & \multirow{2}{*}{$\mathbf{Z}$} \\
\hline & Media & D. E. & $\mathbf{M}$ & D. E. & \\
\hline $\begin{array}{l}\text { PSS - 1 } \\
\text { (Factor S) }\end{array}$ & $-2,39$ & 5,64 & $-4,74$ & 6,17 & $3,91 * * *$ \\
\hline $\begin{array}{l}\text { PSS - } 2 \\
\text { (Factor N) }\end{array}$ & 2,36 & 14,94 & $-3,49$ & 16,48 & $3,68 * * *$ \\
\hline PSS Total & $-0,03$ & 17,97 & $-8,23$ & 20,04 & $3,43 * *$ \\
\hline
\end{tabular}

En la tabla 19, podemos apreciar la existencia de diferencias significativas en el factor $\mathrm{S}$ entre los choferes que manifiestan tener problemas familiares respecto de los que no los presentan, a favor de los segundos; asimismo, se han encontrado diferencias significativas en el factor $\mathrm{N}$ entre los conductores que indican tener problemas familiares respecto de los que no los presentan, a favor de los segundos. 
Finalmente, se han encontrado diferencias estadísticamente significativas entre los estilos de comportamiento en situaciones de tráfico entre los choferes que manifiestan tener problemas familiares respecto de los choferes que no los presentan, a favor de los segundos.

\section{Análisis cualitativo}

Tabla 20. Estilos de comportamiento de los choferes en situaciones de tráfico.

\begin{tabular}{llll}
\hline & N observado & N esperado & Residual \\
\hline Tipo N & 306 & 246,7 & 59,3 \\
Tipo I & 148 & 246,7 & $-98,7$ \\
Tipo S & 286 & 246,7 & 39,3 \\
\hline Total & 740 & & \\
\hline Chi-cuadrado & 60,011 & & \\
Gl & 2 & & \\
Sig. asintót. &, 000 & & \\
\hline
\end{tabular}

En la tabla 20, podemos apreciar que en el grupo de choferes particulares y de servicio público examinados predomina el tipo $\mathrm{N}$, es decir conductores cuya conducta es desajustada, de alto riesgo y peligrosa.

Tabla 21. Estilos de comportamiento según vehículo particular o de servicio público.

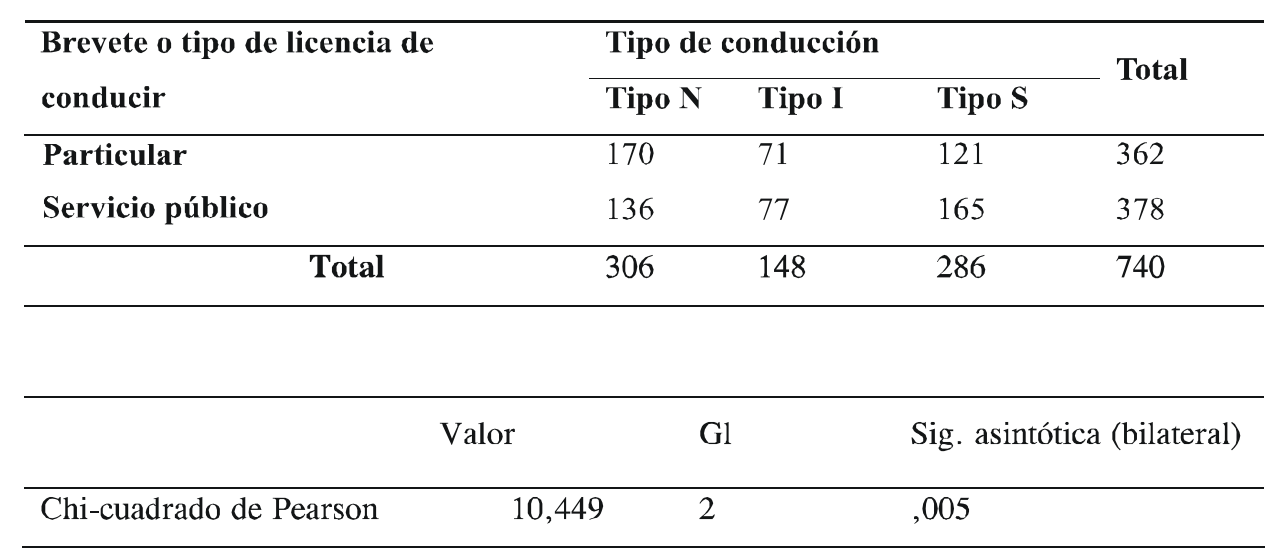

En la tabla 21, encontramos que el estilo de comportamiento tipo N, predomina en los choferes particulares, en tanto que el tipo $\mathrm{S}$ predomina en los conductores profesionales de servicio público. 
Tabla 22. Análisis Chi-cuadrado de los estilos de comportamiento por tipo de vehículo particular o de servicio público que conduce.

\begin{tabular}{|c|c|c|c|c|c|}
\hline \multirow{2}{*}{$\begin{array}{l}\text { Tipo de automóvil que } \\
\text { Conduce }\end{array}$} & \multicolumn{4}{|c|}{ Tipo de conducción } & \multirow{2}{*}{ Total } \\
\hline & Tipo N & & Tipo I & Tipo $\mathbf{S}$ & \\
\hline Particular & 107 & & 51 & 91 & 249 \\
\hline Taxi & 65 & & 33 & 54 & 152 \\
\hline Ómnibus urbano & 99 & & 49 & 80 & 228 \\
\hline Ómnibus interprovincial & 35 & & 15 & 61 & 111 \\
\hline \multirow[t]{2}{*}{ Total } & 306 & & 148 & 286 & 740 \\
\hline & Valor & Gl & & \multicolumn{2}{|c|}{ Sig. asintotica (bilateral) } \\
\hline Chi-cuadrado de Pearson & 14,949 & 6 & & 021 & \\
\hline
\end{tabular}

En la tabla 22, se observa que el tipo $\mathrm{N}$ es el predominante en los choferes particulares; taxistas y microbuseros, en tanto que el factor $\mathrm{S}$, es el predominante en los choferes interprovinciales.

Tabla 23. Análisis Chi-cuadrado de los tipos de conducción por edad cronológica.

\begin{tabular}{ccllll}
\hline \multirow{2}{*}{ Edad cronológica } & \multicolumn{2}{l}{ Tipo de conducción } & \multirow{2}{*}{ Total } \\
\cline { 2 - 5 } & Tipo N & Tipo I & Tipo S & \\
\hline menos a 19 & 2 & 0 & 2 & 4 \\
$\mathbf{2 0}$ & $\mathbf{2 9}$ & 64 & 24 & 31 & 119 \\
\hline $\mathbf{3 0}$ & $\mathbf{3 9}$ & 91 & 46 & 93 & 230 \\
$\mathbf{4 0}$ & $\mathbf{4 9}$ & 92 & 45 & 85 & 222 \\
\hline $\mathbf{5 0}$ & $\mathbf{5 9}$ & 47 & 24 & 56 & 127 \\
$\mathbf{6 0}$ & $\mathbf{9}$ mas & 305 & 8 & 16 & 33 \\
\hline & Total & & 147 & 283 & 735 \\
\hline & & & & & \\
\hline
\end{tabular}

En la tabla 23, se puede observar que no existe una predominancia de estilo de conducta en contextos de tráfico en los sujetos de 19 años; entre los 20-29, se aprecia una predominancia del patrón N; entre los 30-39, se detecta un equilibrio de los estilos de comportamiento S y 
$\mathrm{N}$; entre los 40-49, se observa un ligera predominancia del patrón N; entre los 50-59, se encuentra una predominancia del factor $\mathrm{S}$, la cual también se va a encontrar entre los choferes de 60 ó más años.

Tabla 24. Análisis Chi-cuadrado de los tipos de conducción por estado civil.

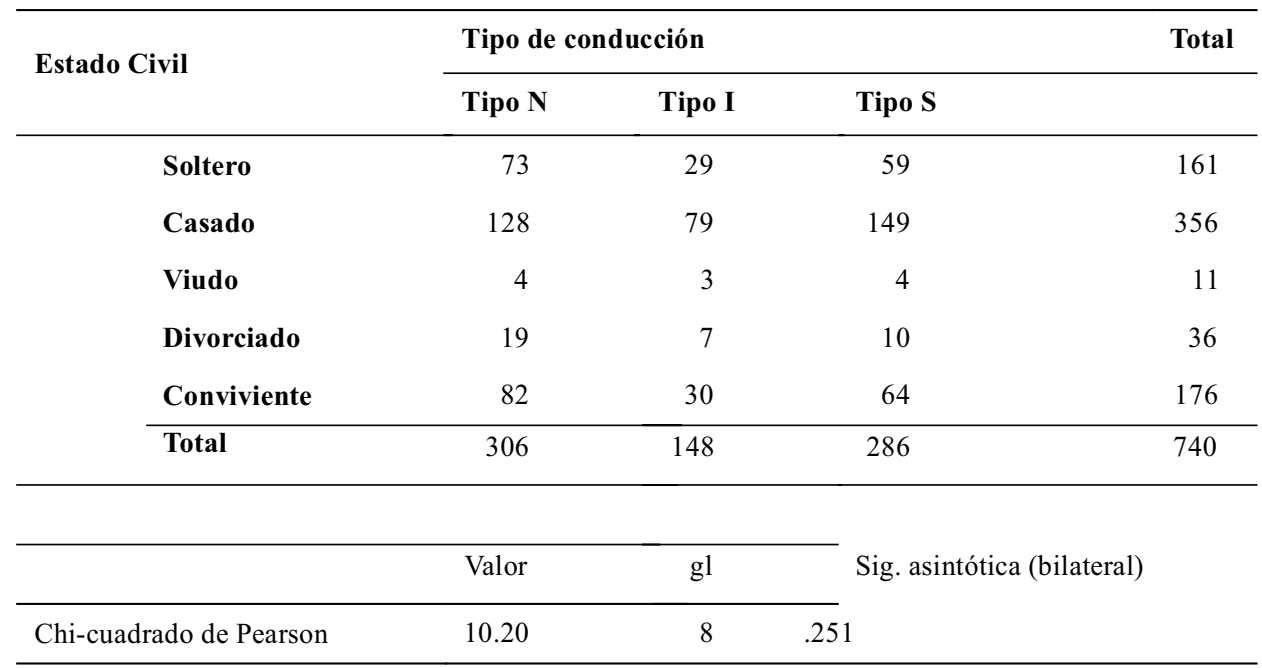

En la tabla 24, podemos apreciar que el tipo $\mathrm{N}$ es predominante en los solteros; en los divorciados y en los convivientes, en tanto que, el tipo $\mathrm{S}$ es el predominante en los casados.

Finalmente, existe un equilibrio en los estilos de conducta $\mathrm{S}$ y $\mathrm{N}$ en los viudos.

Tabla 25. Análisis de los estilos de comportamiento según el grado de instrucción.

\begin{tabular}{lcccc}
\hline \multirow{2}{*}{ Grado de Instrucción } & \multicolumn{2}{c}{ Tipo de conducción } & \multirow{2}{*}{ Total } \\
\cline { 2 - 4 } & Tipo N & Tipo I & Tipo S & \\
\cline { 2 - 5 } Primaria & 14 & 6 & 17 & 37 \\
Secundaria & 155 & 68 & 131 & 354 \\
Superior & 99 & 63 & 104 & 266 \\
Técnica & 35 & 11 & 34 & 80 \\
\hline Total & 303 & 148 & 286 & 737 \\
\hline & Valor & Gl & Sig. asintótica (bilateral) \\
\hline Chi-cuadrado de Pearson & 6.623 & 6 & .357 & \\
\hline
\end{tabular}

En la tabla 25, podemos observar que en los choferes con educación secundaria es predominante el factor $\mathrm{N}$, en los conductores con educación superior es predominante el 
factor $\mathrm{S}$, existiendo un equilibrio de los factores $\mathrm{S}$ y $\mathrm{N}$ en los conductores con educación primaria y técnica.

Tabla 26 Análisis Chi-cuadrado de los estilos de comportamiento en situaciones de tráfico por pertenencia del vehículo.

\begin{tabular}{|c|c|c|c|c|}
\hline & \multicolumn{2}{|c|}{ Tipo de conducción } & \multirow[b]{2}{*}{ Tipo S } & \multirow[t]{2}{*}{ Total } \\
\hline Pertenencia del vehículo & Tipo N & Tipo I & & \\
\hline Propio & 154 & 71 & 125 & 350 \\
\hline Alquilado & 101 & 55 & 71 & 227 \\
\hline Empresa & 51 & 22 & 90 & 163 \\
\hline \multirow[t]{2}{*}{ Total } & 306 & 148 & 286 & 740 \\
\hline & Valor & Gl & \multicolumn{2}{|c|}{ Sig. asintótica (bilateral) } \\
\hline \multicolumn{2}{|c|}{ Chi-cuadrado de Pearson } & 26,163 & 4 & \\
\hline
\end{tabular}

En la tabla 26, se detecta que los choferes que manejan su vehículo propio, así como los que conducen vehículos alquilados, presentan como predominante el tipo $\mathrm{N}$; en tanto que en los choferes que trabajan para empresas predomina el tipo S.

Tabla 27. Análisis de los estilos de comportamiento según el tiempo en la conducción.

\begin{tabular}{|c|c|c|c|c|}
\hline \multirow{2}{*}{ Tiempo en la conducción } & \multicolumn{3}{|c|}{ Tipo de conducción } & \multirow{2}{*}{ Total } \\
\hline & Tipo N & Tipo I & Tipo $\mathrm{S}$ & \\
\hline 1-5 años & 99 & 46 & 66 & 211 \\
\hline 6-10 años & 94 & 38 & 68 & 200 \\
\hline 11-15 años & 50 & 12 & 49 & 111 \\
\hline 16-20 años & 31 & 17 & 37 & 85 \\
\hline 21 a más años & 32 & 35 & 66 & 133 \\
\hline Total & 306 & 148 & 286 & 740 \\
\hline
\end{tabular}

\begin{tabular}{llll}
\hline & Valor & Gl & Sig.asintótica bilateral \\
\hline Chi-cuadrado de Pearson & 30,998 & 8 &, 000 \\
\hline
\end{tabular}

En la tabla 27, se aprecia que el tipo $\mathrm{N}$ predomina entre los conductores que manejan de 1-5; de 6-10, presentándose un equilibrio entre $S$ y $N$ entre los que manejan de 11 a 15, en 
tanto que el tipo $\mathrm{S}$ es predominante en los choferes que manejan de $16-20$ y de 21 a más años.

Tabla 28. Análisis Chi-cuadrado de los tipos de conducción por problemas de salud.

\begin{tabular}{|c|c|c|c|c|}
\hline \multirow{2}{*}{ Problema de salud } & \multicolumn{3}{|c|}{ Tipo de conducción } & \multirow{2}{*}{ Total } \\
\hline & Tipo N & Tipo I & Tipo $\mathbf{S}$ & \\
\hline No & 242 & 127 & 234 & 603 \\
\hline \multirow{3}{*}{ Total } & 64 & 21 & 52 & 137 \\
\hline & 306 & 148 & 286 & 740 \\
\hline & Valor & g1 & & $\begin{array}{l}\text { Sig. asintótica } \\
\text { (bilateral) }\end{array}$ \\
\hline Chi-cuadrado de Pearson & 3,03 & 2 & & 220 \\
\hline
\end{tabular}

En la tabla 28, podemos apreciar que el tipo $\mathrm{S}$ es el predominante entre los choferes que no presentan problemas de salud, pero el estilo de conducta $\mathrm{N}$ es el predominante entre los conductores que presentan problemas de salud.

Tabla 29. Análisis de los estilos de comportamiento según presencia de problemas visuales.

\begin{tabular}{|c|c|c|c|c|c|}
\hline \multirow[b]{2}{*}{ Problema visual } & \multicolumn{4}{|c|}{ Tipo de conducción } & \multirow{2}{*}{ Total } \\
\hline & Tipo N & & Tipo I & Tipo $\mathbf{S}$ & \\
\hline No & 215 & \multicolumn{2}{|r|}{122} & 223 & 560 \\
\hline Sí & 91 & \multicolumn{2}{|r|}{26} & 63 & 180 \\
\hline \multirow[t]{2}{*}{ Total } & 306 & \multicolumn{2}{|r|}{148} & 286 & 740 \\
\hline & & Valor & & \multicolumn{2}{|c|}{ Sig. asintótica (bilateral) } \\
\hline \multicolumn{2}{|c|}{ Chi-cuadrado de Pearson } & 9,363 & & \multicolumn{2}{|c|}{,009 } \\
\hline
\end{tabular}

En la tabla 29, se puede apreciar que en los choferes que presentan problemas visuales es predominante el tipo $\mathrm{N}$, en tanto que existe un relativo equilibrio de los factores $\mathrm{S}$ y $\mathrm{N}$ entre los choferes que no presentan dichos problemas, con una cierta tendencia a $S$. 
Tabla 30. Análisis de los estilos de comportamiento según problemas familiares.

\begin{tabular}{|c|c|c|c|c|c|}
\hline \multirow[b]{2}{*}{ Problema Familiar } & \multicolumn{4}{|c|}{ Tipo de conducción } & \multirow[b]{2}{*}{ Total } \\
\hline & Tipo N & & Tipo I & Tipo S & \\
\hline No & 248 & & 131 & 255 & 634 \\
\hline Sí & 58 & & 17 & 31 & 106 \\
\hline \multirow[t]{2}{*}{ Total } & 306 & & 148 & 286 & 740 \\
\hline & & Valor & Gl & \multicolumn{2}{|c|}{ Sig. asintótica (bilateral) } \\
\hline \multicolumn{2}{|c|}{ Chi-cuadrado de Pearson } & 9,147 & 2 & \multicolumn{2}{|c|}{010} \\
\hline
\end{tabular}

En la tabla 30, se aprecia que en los choferes que manifiestan problemas familiares es predominante el factor $\mathrm{N}$, observándose un relativo equilibrio de los tipos $\mathrm{S}$ y $\mathrm{N}$ en los conductores que no presentan problemas familiares, tendiendo más bien a $\mathrm{S}$.

\section{CONCLUSIONES}

Los resultados obtenidos en el presente estudio nos han permitido llegar a las siguientes conclusiones:

1. Los conductores de servicio público (taxistas, microbuseros y choferes interprovinciales), y los automovilistas particulares de Lima Metropolitana presentan patrones de conducta diferenciados en contextos de tráfico. En tal sentido, hemos hallado diferencias significativas a nivel global $(Z=-3.83)$ entre ambos grupos, a favor de los choferes de servicio público, los cuales revelan que los primeros son seguros; prudentes; responsables; atentos y precavidos, los cual estaría ratificando la hipótesis general.

2. Se han encontrado diferencias estadísticamente significativas en los estilos de comportamiento globales en contextos de tráfico entre los automovilistas particulares y los choferes interprovinciales, a favor de los segundos, lo cual estaría ratificando la hipótesis específica 3.

3. Se han detectado diferencias significativas en los patrones de conducta globales en contextos de tráfico entre los microbuseros y los choferes interprovinciales, a favor del segundo grupo, lo cual estaría ratificando la hipótesis específica 5.

4. Se observan diferencias estadísticamente significativas en los estilos de comportamiento globales en contextos de tráfico entre los taxistas y los choferes interprovinciales, a favor de los segundos, lo cual estaría ratificando la hipótesis específica 6.

En suma, las conclusiones 2, 3 y 4, nos revelan que los choferes interprovinciales presentan comparativamente estilos de comportamiento positivos en contextos de tráfico que le permiten conducir bajo una percepción y aceptación del riesgo adecuada; son precavidos, atentos y responsables, con alto nivel de seguridad y controlan adecuadamente su conducta y constituye el grupo de choferes que marca la diferencia con las otras 
modalidades examinadas (particulares; microbuseros y taxistas), que presentan una conducta desajustada, peligrosa y de alto riesgo.

Este hallazgo aclara la primera conclusión en el sentido de que no todos los choferes de servicio público como es el caso de los taxistas y microbuseros tienen adecuados estilos de comportamiento en contextos de tráfico. Tan es así que los valores negativos obtenidos por estos grupos en el instrumento se eliminan e inclusive se hacen positivos cuando se suma a estos el grupo de choferes interprovinciales. Además, el otro hallazgo importante es que los automovilistas particulares también presentan valores negativos en sus patrones de conducta en contextos de tráfico.

Por otra parte, el análisis cualitativo nos permite apreciar que en la muestra examinada de choferes de servicio público y particulares, predomina el estilo de conducta tipo $\mathrm{N}$, es decir conductores cuya conducta es desajustada, de alto riesgo y peligrosa.

Asimismo, encontramos que el estilo de comportamiento tipo N, predomina en los choferes particulares, en tanto que el tipo $\mathrm{S}$, es predominante en los conductores de servicio público. Finalmente, detectamos que el tipo $\mathrm{N}$ es el predominante en los automovilistas particulares; taxistas y microbuseros, en tanto que el factor $\mathrm{S}$, es el predominante en los choferes interprovinciales.

5. Se han detectado diferencias estadísticamente significativas en los estilos de comportamiento globales en contextos de tráfico en razón a la variable pertenencia del vehículo, entre los choferes que conducen su vehículo propio y los que conducen vehículos de propiedad de una Empresa, a favor de los segundos; así como entre los que conducen vehículos alquilados y los que conducen vehículos de propiedad de una Empresa, a favor del segundo grupo.

La conclusión 5, nos revela que los choferes que manejan vehículos de propiedad de una Empresa presentan estilos positivos de conducta en contextos de tráfico frente a los que son propietarios o a los que alquilan vehículos.

El análisis cualitativo nos indica que en los choferes que manejan su propio vehículo, así como los que conducen vehículos alquilados, es predominante el tipo $\mathrm{N}$; en tanto que en los choferes que trabajan para Empresas predomina el tipo S.

6. Se han detectado diferencias significativas en los estilos de conducta globales en contextos de tráfico en razón a la variable tiempo en la conducción, entre los que conducen entre 1-5 años, respecto de los que conducen entre los 16-20 años, a favor de los segundos; asimismo, hemos encontrado diferencias entre los que manejan entre 15 años, respecto de los que conducen 21 o más años; del mismo modo, se hallan diferencias entre los choferes que manejan de 6-10 años, respecto de los que lo hacen 21 o más años; por último, se hallan diferencias entre los que conducen entre 11-15 años, respecto de los que manejan 21 o más años. En suma, a mayor tiempo en la conducción van mejorando gradualmente los estilos de comportamiento en contextos de tráfico.

En el análisis cualitativo se registra que el tipo $\mathrm{N}$ predomina entre los conductores que manejan de 1-5 y de 6-10 años, presentándose un equilibrio entre $\mathrm{S}$ y $\mathrm{N}$ entre los que manejan entre 11-15 años, en tanto que el tipo $S$ es predominante en los choferes que manejan de 16-20 y de 21 o más años. 
7. Se registran diferencias significativas en los estilos de comportamiento globales en contextos de tráfico en razón a la variable frecuencia de accidentes de tránsito, entre los que nunca han tenido accidentes respecto de los que han presentado un accidente; asimismo, entre los que nunca han tenido accidentes respecto de los que han presentado dos accidentes; entre los que nunca han tenido accidentes respecto de los que han tenido tres o más accidentes; $y$, finalmente entre los que han presentado dos respecto de los que han tenido más de tres accidentes. En síntesis, se aprecia que la frecuencia de accidentes de tránsito en choferes nos revela estilos de comportamiento negativos e inadecuados en contextos de tráfico que se van agudizando con el tiempo, frente a la ausencia de accidentes de grupos de choferes que se expresa en una adecuada conducción.

8. Se aprecia la existencia de diferencias significativas en los estilos de comportamiento globales en contextos de tráfico en razón a la variable problemas familiares, entre los choferes que manifiestan tener problemas familiares, respecto de los automovilistas que no reportan este tipo de conflicto, a favor de los segundos.

El análisis cualitativo pone de manifiesto que en los choferes que manifiestan problemas familiares es predominante el factor $\mathrm{N}$, observándose un relativo equilibrio entre los tipos $\mathrm{S}$ y $\mathrm{N}$ en los conductores que no refieren dichos problemas.

9. Se registran diferencias significativas en el estilo de comportamiento específico $S$, en contextos de tráfico, en razón a la variable problemas de salud, entre los conductores que manifiestan tener problemas de salud, respecto de los choferes que no reportan este tipo de problemas, a favor de los segundos, hallazgo que nos indica que la buena salud guarda una relación favorable con el estilo $\mathrm{S}$ de conducción positiva.

10. Se detectan diferencias significativas en el patrón de conducta específico $\mathrm{N}$, en contextos de tráfico, en razón a la variable problemas de motricidad, entre los choferes que manifiestan este tipo de problemas, respecto de los conductores que reportan no presentarlos, a favor del segundo grupo, lo cual indica que la existencia de problemas motrices genera irritabilidad, agresividad, intolerancia, conducta riesgosa y antisocial en la conducción.

11. El análisis cualitativo efectuado en los estilos de conducta en situaciones de tráfico y la edad cronológica nos indica que en los choferes de 19 años no existe un patrón predominante; entre los 20-29 se aprecia una predominancia del patrón N; entre los 30-39 y 40-49 se observa un equilibrio de los estilos S y N; entre los 50-59 se aprecia una predominancia del factor $\mathrm{S}$, la cual también se va a observar entre los choferes de 60 ó más años. En suma, a mayor edad cronológica mejora el estilo de comportamiento en contextos de tráfico y viceversa.

12. El análisis cualitativo realizado en los patrones de comportamiento en situaciones de tráfico y el estado civil, nos indica que el tipo $\mathrm{N}$ es predominante en los solteros; en los divorciados y en los convivientes, en tanto que el tipo $\mathrm{S}$ es el predominante en los casados. Finalmente, existe un equilibrio en los estilos de conducta $\mathrm{S}$ y $\mathrm{N}$ en los viudos.

13. El análisis cualitativo efectuado en los estilos de comportamiento en situaciones de tráfico y el grado de instrucción, nos revela que en los choferes de educación secundaria es predominante el factor $\mathrm{N}$; en los conductores con educación superior es predominante 
el factor $\mathrm{S}$, existiendo un equilibrio de los factores $\mathrm{S}$ y $\mathrm{N}$ en los conductores con educación primaria y técnica.

14. El análisis cualitativo efectuado en los estilos de conducta en situaciones de tráfico y la conducción como actividad principal, nos indica que los automovilistas cuya conducción no es su ocupación principal, predomina el tipo $\mathrm{N}$, en tanto que los choferes que manifiestan que la conducción es su actividad principal, encontramos un equilibrio entre los estilos $\mathrm{S}$ y $\mathrm{N}$.

15. El análisis cualitativo realizado en los patrones de comportamiento en situaciones de tráfico y la presencia de problemas visuales nos indica que en los choferes que refieren problemas visuales es predominante el tipo $\mathrm{N}$, en tanto que existe un relativo equilibrio entre los estilos $\mathrm{S}$ y $\mathrm{N}$ en los conductores que no manifiestan dichos problemas.

\section{Sugerencias}

1. Con el propósito de conocer esta problemática a nivel nacional, se deben promover, no sólo estudios de replicación de la presente investigación en otros departamentos del Perú, sino la realización de otros estudios donde se consideren otras modalidades de conducción como: mototaxistas; movilidad escolar; motociclistas; ciclistas, entre otros, así como también la inclusión de otras temáticas relacionadas con el tráfico y la seguridad vial tales como: el estrés; las conductas antisociales; la satisfacción familiar; las habilidades sociales, etc., de los choferes y que nos permitan a mediano plazo tener un perfil del conductor en cada una de las modalidades.

2. Se debe propiciar la realización de estudios interdisciplinarios que incluyan reportes médicos (de tipo: forense; oftalmológico; traumatológico, entre otros); información policial; estudios de la sociología; de trabajo social; de la pedagogía, etc. que puedan darle una mayor cobertura de acción a los hallazgos, que permitan a corto plazo formular un plan de educación vial a nivel nacional.

\section{REFERENCIAS BIBLIOGRÁFICAS}

1. Álvarez, F.J. (1996). Seguridad vial y medicina de tráfico. Barcelona: Masson.

2. Buela-Casal, G. y MIRÓ, H. (1994). «Conocimiento y actitudes hacia el código de circulación y regulación de la conducta». Revista Mapfre Seguridad, 54, Segundo Trimestre, 11-19.

3. Caparros, A. (1985). «Aspectos históricos de la psicología aplicada a la conducción». I Reunión Internacional de Psicología del Tráfico y Seguridad Vial. Valencia. DGT. 39.

4. Chuliá, V. (1995). «La atención a las víctimas de los accidentes de tráfico». En Montoso, L; Carbonell, E; Sanmartín, J. y Tortosa, F. (Edit.): Seguridad vial: Del factor humano a las nuevas tecnologías. Madrid: Síntesis.

5. De La Fuente, J.L. (1995). La seguridad activa y pasiva en el vehículo. Madrid: Dossat 2000.

6. Fricke, L.B. (1990). Traffic accident reconstruction. Northestern University Traffic Institute. 
7. Germain, J.(1996). «El psicólogo y la seguridad vial». Revista de Psicología General y Aplicada. XXI 85-889.

8. Monterde, H.(1987). «La evaluación psicológica por medio del ordenador». Validez del constructo y fiabilidad de las actuales pruebas psicológicas para conductores basadas en ordenador. Psicología del trabajo y de las organizaciones 6.

9. Lasen, M.(1995). «La investigación de los accidentes de tráfico». En Montoso,L; Carbonell, E; Sanmartín, J. y Tortosa, F. (Ed.): Seguridad Vial: Del factor humano a las nuevas tecnologías. Madrid: Síntesis.

10. Lillo, J. (1995). «Diseño ergonómico del vehículo». En Montoro,L; Carbonell,E.; Sanmartín, J. y Tortosa, F. (Edit.): Seguridad vial: Del factor humano a las nuevas tecnologías. Madrid: Síntesis.

11. Montoro Gonzales, L.(1996). «El futuro de la actividad investigadora en materia de trafico y seguridad vial». Inst. International Congress on Traffic and Road Safety in the Basque Country. Vitoria, 7-9 de octubre de 1996.

12. Montoro Gonzales, L. (1998). «Retos de futuro en el ámbito del tráfico, el transporte y la seguridad vial». Instituto Universitario de Tráfico y Seguridad Vial (INTRAS). Papeles del psicólogo, 1998. N 70. pp. 17-23.

13. Montoro, L.; Soler, J.; Tortosa, F. «El examen psicotécnico». Revista de Tráfico. Año IV $\mathrm{N}^{\circ} 29$, enero 88.

14. Montoro, L.; Carbonell, E. y Monterde, H. (1990). «El reconocimiento psicológico de los conductores: propuesta para una nueva normativa». Ponencia en el IV Congreso Nacional de Centros de Reconocimiento de Conductores para la Seguridad Vial. RECOSEVIAL IV. Salamanca, octubre de 1990.

15. Montoro, L.; Tejero, P. y Bañuls, R. (1994). «Reciclaje y formación permanente: una exigencia de futuro para los conductores, los profesores de autoescuelas y los examinadores». Ponencia en el XXIV Congreso Nacional de Autoescuelas. Valencia, 7-10 de diciembre de 1994. 